\title{
Développement de la confiance entre partenaires de joint ventures internationales: les mécanismes à succès
}

\author{
Elie Virgile Chrysostome 1 \\ Université de Moncton
}

\section{Introduction}

La confiance est un problème qui préoccupe quotidiennement les gens des milieux d'affaires. Elle ne rate presque aucun de leurs rendez-vous et les hante en permanence. Si tout le monde reconnaît la nécessité de la confiance, en revanche tout le monde ne semble pas prêt à faire les sacrifices nécessaires pour créer et développer cette confiance. Chacun a peur qu'en faisant des sacrifices, il ne donne à son partenaire l'occasion d'utiliser ces sacrifices à ses dépens, pour finalement l'exploiter et le dominer. Il résulte de cette situation que, par crainte d'être perdant, chaque partenaire de joint venture reste sur ses gardes et a tendance à garder ses atouts pour ses seuls intérêts personnels, ou à tout le moins, à limiter la jouissance que son allié pourrait en tirer. Pourtant, il est évident que sans la confiance, aucune sorte de joint venture ne peut se développer et réussir de manière durable. L'objectif de cet article est de proposer un processus de création de la confiance dans les joint ventures internationales.

\section{Confiance dans les joint ventures internationales : un ingrédient essentiel}

\section{Qu'est-ce que la confiance?}

La confiance est comme un mode de contrôle informel qui gouverne des acteurs mutuellement identifiés. Elle permet de réduire l'incertitude relative au comportement mutuel par un processus d'auto-contrôle. C'est un mode de contrôle doté de souplesse ${ }^{2}$ et qui, en tant que tel, permet de prendre des initiatives en dehors des règles existantes ainsi que de développer une longanimité mutuelle propice à la stabilité des relations. La confiance est un processus qui met en oeuvre des anticipations et des obligations entre deux parties. C'est un processus dans lequel celui qui fait confiance a des anticipations quant au comportement de l'autre, dans les domaines d'intérêt mutuel, et où l'autre à qui on fait confiance se sent obligé de se conformer à ces anticipations. La confiance est un complément nécessaire et reste un ingrédient fondamental du succès des alliances. C'est une importante énergie de maintenance des joint ventures internationales.

\section{Importance de la confiance au sein des joint ventures internationales}

L'intérêt de la confiance dans les joint ventures se justifie essentiellement par les insuffisances constatées au sein des principaux paradigmes d'alliance expérimentés jusqu'à maintenant. En effet, ni le paradigme de la dépendance des ressources ni celui des coûts de transaction n'ont réussi à se mettre à l'abri des divers problèmes de dysfonctionnement, bien souvent liés aux pesanteurs du formalisme des contrats et aux comportements opportunistes des partenaires. En d'autres termes, ces deux types de paradigmes d'alliance trébuchent à cause du manque de confiance entre les partenaires. Ainsi, le paradigme de la dépendance des ressources qui préfère un mode de contrôle formel n'offre pas aux alliés la souplesse nécessaire pour leur permettre de dépasser les règles du contrat, à chaque fois qu'un tel dépassement est indispensable pour résoudre des problèmes qui apparaissent au sein de l'alliance. Le paradigme de la dépendance des ressources postule que l'organisation identifie au sein de son environnement des dépendances dont elle cherche à s'affranchir au moyen des initiatives comme l'alliance. Par conséquent, un tel paradigme n'accorde pas beaucoup d'importance à la logique supra organisationnelle (logique informelle), alors que celle-ci est pourtant nécessaire au succès de l'alliance. L'absence de cette logique supra organisationnelle, à savoir la confiance, est un facteur sérieux de dysfonctionnement et une source de conflits au sein des joint ventures 
internationales. Le paradigme des coûts de transaction quant à lui, en se focalisant sur les transactions et les structures de gestion, procède d'une vision statique des alliances et ignore lui aussi le rôle des processus informels. Ce faisant, le paradigme des coûts de transaction ignore le rôle de la confiance au sein des partenariats et ouvre la voie à l'adoption de comportements opportunistes par les partenaires. Ces comportements opportunistes sont eux-mêmes une source de conflits entre les alliés. Or, la confiance permet d'éviter ces comportements opportunistes et les conflits qui en découlent.

Les témoignages de chercheurs et de chefs d'entreprises célèbres fusent sur l'importance et la nécessité de la confiance dans les alliances. À titre d'exemple, Gene Slowinski, directeur des Études sur les Alliances à l'Université Rutgers (New Jersey, ÉU) a affirmé il y a quelques années que «un assaut implacable de la confiance et $d u$ respect est un facteur capital dans le fonctionnement des alliances ...». Cette perception de la confiance est partagée par Peter Booth qui était viceprésident de la Stratégie du développement de Corning inc. lorsqu'il a affirmé que «la confiance est l'ingrédient le plus important en business....». La confiance est le premier des mécanismes informels de contrôle dans les joint ventures internationales et aucune autre variable n'influence de manière aussi approfondie les relations interorganisationnelles. À ce titre, la confiance constitue un élément clé dans l'émergence et la réussite d'une joint venture internationale car cette dernière implique des risques de pertes de ressources pouvant se réaliser en cas de comportement non-coopératif d'un partenaire. La confiance protège dans l'espace et le temps l'engagement des partenaires de la joint venture et sa disparition est plus grave que le non-respect d'une règle de contrat. Cette importance de la confiance est illustrée par une phrase assez célèbre du président de la firme américaine «Dow Chemical» lorsque celui-ci, en parlant de l'alliance entre sa compagnie et la firme «Personal Care», a déclaré il y a quelques années: "les contrats sont maintenant vieux de nombreuses années; nous ne les regardons plus beaucoup...». De même, en commentant l'alliance de la firme «Schlumberger» avec la firme «Dow Chemical», le vice-président exécutif de cette dernière déclara ceci: "Nous ne sommes pas seulement des partenaires, nous sommes de bons amis. Et nous prenons en compte tous les aspects qui concernent les besoins de chacun. Nous pouvons nous battre comme des chats et chiens sur une chose donnée, mais quand il s'agit de grandes questions de politique, nous sommes comme des partenaires et amis» ${ }^{3}$. Ces déclarations faites par des gestionnaires très expérimentés prouvent toute l'importance et la nécessité de la confiance dans les joint ventures internationales.

Au total, on peut dire que l'importance de la confiance dans les joint ventures internationales repose sur le fait qu'elle permet d'éviter les conflits entre les partenaires et d'assurer le succès de leur projet de coopération grâce à un développement de la dimension sociale, c'est-à-dire entre autres, des relations interpersonnelles entre les partenaires de joint ventures.

\section{Processus de création de la confiance dans les joint ventures internationales}

Le processus de création de la confiance est d'ordinaire un processus très difficile et de longue haleine. Il est particulièrement plus délicat en matière de partenariats internationaux car les intérêts en jeu dans de pareilles circonstances sont très élevés et quelques fois divergents. Le modèle proposé dans la présente recherche (figure No.1) comprend deux phases principales, à savoir celle de la vérification des mécanismes de base et celle du déploiement des mécanismes procéduraux :

\section{La vérification des mécanismes de base}

Les mécanismes de base sont ceux qui favorisent l'émergence d'une confiance ex ante. Cette confiance est le point de départ de tout projet de coopération interentreprises et, à ce titre, elle est indispensable pour toute coopération à long terme comme les joint ventures internationales. En effet, l'aboutissement normal du déploiement des mécanismes de la confiance ex ante (mécanismes de base) dans un projet international est le choix de partenaires appropriés. Ces mécanismes sont:

\section{L'adhésion à des normes sociales partagées}

L'adhésion à des normes sociales partagées favorise, en l'absence de règles formalisées, une confiance liée à l'insertion dans des réseaux de relations interpersonnelles. En effet, l'adhésion à des normes sociales partagées crée entre les partenaires potentiels d'une joint venture une véritable proximité sociale. Ces partenaires potentiels appartiennent désormais à un même univers de jugement en partageant certaines valeurs clés. De ce point de vue, ils peuvent anticiper les comportements les uns des autres sans que ces comportements ne soient 
prédéfinis par des règles préalables. Cette possibilité d'anticipation réduit l'incertitude pouvant survenir au sein d'une future coopération, ce qui réduit par ce fait même l'éventualité de comportements opportunistes des futurs partenaires et favorise entre ces derniers l'établissement spontané de la confiance.

\section{La reconnaissance des compétences du partenaire potentiel}

La confiance ex ante repose entre autres sur des signaux de compétence, et cette dernière constitue la première dimension de la confiance. En effet, la recherche d'avantages liés aux compétences du partenaire étant l'une des principales raisons justifiant la création d'un partenariat, une entreprise désirant s'engager dans une joint venture aura tendance à faire confiance à un partenaire potentiel si elle est convaincue que ce partenaire possède les compétences qu'elle recherche. Pour l'entreprise désireuse de s'engager dans la création d'une joint venture, la compétence du partenaire constitue un gage d'efficacité. La reconnaissance des compétences du partenaire potentiel ainsi que des avantages stratégiques qui y sont associés représente une composante incontournable dans l'établissement de la confiance ex ante.

\section{La reconnaissance de la réputation du partenaire potentiel}

La réputation est généralement le fruit d'une bonne performance entretenue de façon durable. Ici, le terme performance ne réfère pas seulement à des considérations quantitatives courantes telles que le profit, la part de marché ou encore la rentabilité, mais recouvre aussi des considérations qualitatives telles que la qualité des produits, l'image de marque ou encore l'exercice d'une responsabilité sociale, entre autres vis-à-vis des normes écologiques. La réputation constitue de ce point de vue un important actif intangible que l'entreprise entretient par des investissements multiformes dans la qualité de ses activités. Les avantages associés à la réputation sont nombreux. Un de ceux-ci et non le moindre est la confiance qu'elle inspire aux partenaires d'affaires. Plus spécifiquement dans le cas des joint ventures internationales, la réputation d'un partenaire potentiel contribue à l'établissement d'un climat de confiance puisqu'elle réduit à certains égards l'incertitude à laquelle les entreprises alliées seraient confrontées dans l'éventualité d'une coopération. Ce climat de confiance sera d'autant plus grand que la réputation du partenaire potentiel découle entre autres de sa participation antérieure à une joint venture réussie.

\section{La conviction de l'efficacité du système judiciaire de l'environnement d'opération}

Le système judiciaire de l'environnement d'opération joue un rôle important dans l'établissement de la confiance entre les partenaires potentiels d'une joint venture internationale. En effet, l'efficacité du système judiciaire contribue à inhiber certaines réticences et craintes des partenaires potentiels car ces derniers savent à l'avance qu'en cas de conflits dont la solution échappe à leur volonté de coopérer, ils peuvent s'en remettre à une justice rapide et impartiale. L'efficacité du système judiciaire réfère ici à une perception positive des partenaires potentiels sur la justice de leur environnement d'opération.

\section{Le déploiement des mécanismes procéduraux}

Les mécanismes procéduraux sont ceux par lesquels les partenaires d'une joint venture apprennent à se faire confiance. À ce titre, ils contribuent activement à un véritable développement de la confiance entre les dits partenaires. La confiance dont il s'agit ici est une confiance procédurale. Ces mécanismes sont:

\section{La mise en ouvre d'une solidarité sociale entre partenaires}

\section{- Le développement des amitiés}

Pour des partenaires qui vont coopérer sur une échelle de longue durée, l'établissement d'amitiés est indispensable afin de leur permettre de mieux se connaitre et de se faire confiance. Le développement de telles amitiés peut se faire grâce à la création de relations personnelles entre les partenaires. Ceci demande beaucoup de temps, car les relations personnelles se développent progressivement grâce à un échange quotidien d'informations et une compréhension mutuelle de plus en plus approfondie. La familiarité et la continuité sont donc indispensables. Au départ, la confiance est souvent une question d'amitié qui existe entre deux personnes et qui va par la suite rejaillir et s'enraciner dans chacune des organisations d'où proviennent ces personnes. Les partenaires de joint ventures doivent prendre $\mathrm{du}$ recul et modérer les énergies qu'ils consacrent à leurs intérêts matériels pour 
accorder un peu plus de temps à leurs amitiés afin de développer la confiance. Pour ce faire, les activités sociales extra professionnelles sont nécessaires.

\section{La transparence}

\section{- La facilitation de la communication}

Chaque partenaire doit favoriser une transparence et une fluidité dans l'échange d'information afin d'inspirer confiance à son allié. Il doit notamment éviter la rétention d'informations en envoyant ses documents pertinents (rapports d'activité et autres) à son allié. En retour, celuici doit prouver qu'il ne prend aucune décision dans son propre intérêt et au détriment de l'autre ou du partenariat. Les rencontre amicales informelles dans le cadre d'événements sociaux ou de loisirs (dîners ou compagnie de plaisance dans un café par exemple) sont indispensables pour faciliter la communication. Elles favorisent non seulement une prédisposition des partenaires à communiquer honnêtement mais parfois un rapprochement qui permet l'échange d'informations précieuses qui n'auraient jamais circulé de façon formelle. Il en est de même des communications informelles au téléphone, par fax ou par courrier électronique qui doivent être encouragées. De tels échanges peuvent comporter des risques mais il s'agit de risques qui méritent d'être pris car, d'une part, ils peuvent être rentables et d'autre part, les partenaires ou travailleurs amis savent en général établir des frontières dans ces échanges.

\section{- L'accès réciproque des travailleurs de la joint venture aux différentes entreprises-mères}

Il s'agit ici de la nécessité pour les travailleurs des firmes alliées, notamment les travailleurs des départements $\mathrm{R} \& \mathrm{D}$, de pouvoir séjourner temporairement dans les laboratoires de leurs homologues afin d'échanger de manière très personnelle des expériences et de développer des savoir-faire privilégiés qui permettront à chaque partenaire de gagner.

\section{La gestion adéquate des spécificités socioculturelles et conflictuelles}

\section{- Une valorisation de la dimension culturelle}

La prise en charge de la culture de chaque partenaire dans la gestion des relations de la joint venture peut servir de fondation pour renforcer et développer la confiance existant entre eux. Il est donc indispensable que chaque partenaire s'efforce de comprendre la culture de gestion de son allié.

\section{- L'anticipation des discordes et suspicions}

Les désaccords sont inhérents aux alliances et les partenaires ne doivent pas attendre que ces désaccords se développent et pourrissent, évidemment avec la conséquence que ceci nuirait au développement de la confiance. Pour éviter cette situation et favoriser un climat de confiance, chaque partenaire doit se préoccuper d'être le premier à anticiper ces désaccords en discutant très ouvertement et très franchement avec son allié. Il doit clairement expliquer à ce dernier les raisons de ses prises de position et lui accorder en retour une oreille attentive. Une telle attitude est particulièrement indispensable pour bâtir la confiance avec les partenaires qui n'aiment pas la confrontation. Il est aussi nécessaire d'éviter un excès de formalisme dans les relations entre partenaires, puisqu'un tel excès constitue une source de suspicions et de méfiance qui peuvent menacer le développement de la confiance.

\section{- La répulsion et la prévention des décisions improvisées}

Les surprises sont très nuisibles au développement de la confiance et doivent être rigoureusement évitées dans le cadre des activités de partenariat. Chaque partenaire doit prendre le soin d'informer et de convaincre son allié chaque fois qu'il envisage de prendre une décision qui pourrait affecter d'une manière ou d'une autre les intérêts de ce dernier. Aussi, la mise en œuvre de toutes les décisions prises conjointement doit suivre un rythme d'exécution qui satisfait tous les partenaires. En effet, les partenaires de joint venture n'ont pas nécessairement la même notion de temps et une mise en œuvre trop rapide et non progressive d'une décision conjointe peut se révéler fatale pour le processus de formation de la confiance.

\section{Le contrôle conjoint de la gestion de la joint venture}

\section{- Une gestion flexible et partagée}

La gestion flexible et partagée de la joint venture peut se faire à travers une délégation de pouvoirs et la promotion d'une autonomie en faveur des cadres des services $\mathrm{R} \& \mathrm{D}$. Lorsqu'une telle décentralisation est conjuguée avec les contacts informels existant entre ces cadreschercheurs, elle facilite des contacts plus profonds ainsi 


\section{Figure $n^{\circ} 1$ : Modèle de développement de la confiance au sein des joint ventures internationales}

\begin{tabular}{|c|c|}
\hline Phase 1 : Vérification des mécanisme de base & $\begin{array}{l}\text { Phase } 2 \text { : Déploiement des mécanismes } \\
\text { procéduraux }\end{array}$ \\
\hline $\begin{array}{l}\text { Adhésion à des normes sociales partagées } \\
\text { Reconnaissance des compétences du partenaire } \\
\text { Reconnaissance de la réputation du partenaire } \\
\text { Efficacité du système judiciaire }\end{array}$ & $\begin{array}{l}\text { Solidarité sociale } \\
\text { Transparence } \\
\text { Gestion des spécificités socioculturelles } \\
\text { Contrôle conjoint de la joint venture }\end{array}$ \\
\hline \multicolumn{2}{|c|}{ CONFIANCE ENTRE PARTENAIRES DE LA JOINT VENTURE } \\
\hline
\end{tabular}

que le développement d'un réseau informel d'experts, un réseau qui profiterait naturellement au partenariat. Une gestion flexible et partagée suppose aussi et surtout un partage du pouvoir de contrôle entre les partenaires, ce qui requiert un réel engagement actif de chaque partenaire au sein de la joint venture. Une gestion flexible et partagée s'oppose par définition à une domination du contrôle de la joint venture par un partenaire, même si l'autre partenaire est très minoritaire. En effet, l'hégémonie d'un partenaire sur les autres constitue une menace certaine à la confiance entre ceux-ci. Le développement de la confiance requiert une relation gagnant-gagnant pour chaque partenaire.

\section{Limites du phénomène de confiance dans les joint ventures internationales}

L'analyse précédemment faite dans la présente recherche sur l'importante nécessité de la confiance entre partenaires de joint ventures internationales ne doit pas faire perdre de vue ses limites. En effet, la confiance toute seule ne suffit pas pour garantir la réussite d'un partenariat international. La confiance reste très certainement un ingrédient fondamental qui peut garantir le succès car elle favorise l'engagement et la foi des partenaires. C'est l'énergie de maintenance qui, en facilitant une gestion quotidienne des conflits et des incertitudes, permet d'assurer une longévité à la joint venture internationale. À ce titre, le manque de confiance fragili- serait les relations du partenariat et exposerait ce dernier à l'éclatement en permanence puisque chaque partenaire adopterait des comportements opportunistes qui seraient de nature à en tirer le maximum d'avantages le plus vite possible, peu importe que cela se fasse au détriment de l'autre. Cependant, tous les privilèges qui viennent d'être indiqués comme étant caractéristiques de la confiance ne suffisent pas à permettre à cette dernière d'assurer toute seule la stabilité nécessaire pour les relations de joint venture. L'établissement de relations d'affaires uniquement basées sur les mécanismes informels de contrôle comme la confiance - peut créer des ambiguïtés de nature à engendrer une instabilité de ces relations. Une confiance sans formalisation peut aboutir à la rupture après de nombreux conflits et il n'est pas raisonnable ni même économique d'accroître les enjeux d'une alliance lorsque celle-ci est fondée uniquement sur une relation informelle comme la confiance. En revanche, l'alliance dans laquelle alternent confiance et formalisation est non seulement d'une grande efficacité du point de vue de l'économie des coûts de transaction mais aussi d'une grande stabilité combinant fermeté et souplesse. La formalisation entérine en quelque sorte la validation des hypothèses ou des anticipations sur le comportement du partenaire.

En résumé, on peut dire que la confiance est indispensable pour une gestion saine et durable des relations entre partenaires de joint venture internationale mais elle ne doit pas faire «cavalier seul». 


\section{Confiance et choix de partenaires de joint ventures internationales: quel ordre de préemption}

La question qui se pose ici est celle de savoir si c'est la confiance qui doit précéder le choix des partenaires d'une joint venture et favoriser sa réussite ou si, à l'inverse, c'est plutôt la mise en oeuvre d'un partenariat qui doit favoriser le développement de relations de confiance entre partenaires? Cette question ressemble un peu à l'histoire de la primogéniture entre l'oeuf et la poule. En vérité, il s'agit d'une question très délicate à laquelle aucun spécialiste de partenariat, aussi prétentieux soit-il, ne peut se targuer de pouvoir répondre efficacement. Cependant, une phrase très sibylline de Simmel $^{4}$ apporte un grand éclairage sur ce problème lorsqu'il affirme ceci: «Celui qui sait tout n'a pas besoin de faire confiance; celui qui ne sait rien ne peut même pas faire confiance». Ce constat de Simmel signifie que lorsque d'une façon ou d'une autre, on connaît un peu son partenaire, on peut lui faire un début de confiance et essayer de réaliser la joint venture avec lui. Le sort de ce début de confiance, c'est-à-dire son développement ou sa destruction, dépendra de la qualité de communication que les partenaires obtiendront. Le constat de Simmel constitue assez souvent la réalité que l'on voit quotidiennement dans le monde des affaires. Ceci ne signifie cependant pas que la réalisation d'un partenariat est impossible lorsqu'on ne connaît pas préalablement son partenaire car il existe des partenariats réussis qui sont pourtant nés de simples contacts informels ou de circonstance. La formation de la confiance est un processus très délicat, difficile et long, et il ne suffit pas d'être en partenariat d'affaires avec un allié depuis des années pour le connaître et lui faire confiance. En d'autres termes, pour revenir à la question posée, à savoir quel est entre la confiance et l'émergence du partenariat, l'élément qui vient en premier pour servir de fondation ou de locomotive à l'autre, la réponse qu'on pourrait être tenté de donner est la suivante: il n'existe pas de conflits de privilège entre la confiance et l'émergence d'un partenariat. Les deux éléments sont importants et interdépendants. De plus, il s'interchangent régulièrement dans leurs rôles respectifs, la confiance favorisant la réussite du partenariat, et inversement le partenariat favorisant à son tour le développement de la confiance.

\section{Conclusion}

Le développement de la confiance est une question de premier plan dans l'émergence et la réussite des joint ventures internationales. La plupart des spécialistes sont unanimes pour reconnaître l'importance du rôle que la confiance joue au sein des joint ventures et certains d'entre eux déplorent même qu'on n'y consacre pas suffisamment de temps pour en valoriser la dimension sociale, dimension qui finalement est encore la seule capable de permettre un développement de la confiance entre les partenaires. En effet, la confiance aide au choix des partenaires de joint ventures et facilite le règlement des conflits qui surviennent. Elle apporte aux partenaires la prédisposition nécessaire pour faire face aux incertitudes qui caractérisent le monde des affaires. Tous ces attributs font de la confiance le premier mécanisme informel de contrôle des joint ventures internationales. Cependant, la confiance est un ingrédient trop fragile et son développement exige de la part des partenaires un investissement énorme d'énergie et d'attention. La confiance au sein des partenaires de joint ventures est comme le ciment qui unit les époux au sein d'un mariage. Quelques-uns des principaux mécanismes qui contribuent au développement de la confiance au sein des joint ventures internationales sont: un développement d'amitié, une communication de bonne qualité, une absence de monopole de décision, une anticipation des conflits et une valorisation de la dimension culturelle de même que l'honnêteté, la responsabilité et l'engagement.

\section{Notes et références}

1 Elie Virgile Chrysostome est professeur de management à la Faculté d'administration de l'Université de Moncton au Nouveau-Brunswick.

2 Koenig, C. et G. Van Wijk (1992). Alliances interentreprises: rôle de la confiance. Paris, Economica, pp. 305 à 325.

3 Les citations ont été tirées de Lewis, J. (1990). Partnerships for profits: structuring and managing alliances. New-York, The Three Press.

4 Simmel, G. (1991). Secret et société secrète. Strasbourg, Circé. 